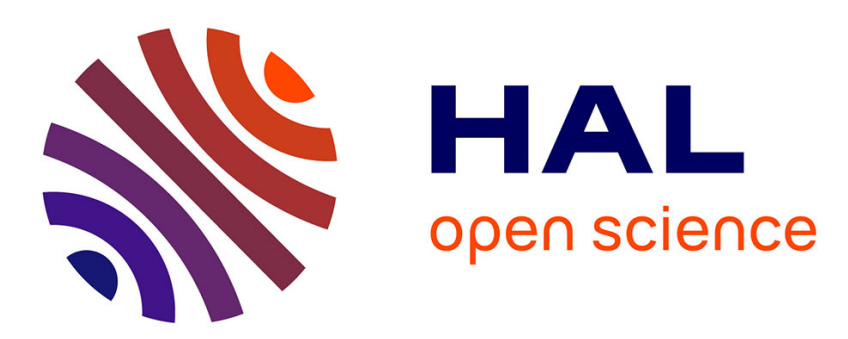

\title{
Optimal energy decay rate for partially damped systems by spectral compensation
}

\author{
Paola Loreti, Bopeng Rao
}

\section{To cite this version:}

Paola Loreti, Bopeng Rao. Optimal energy decay rate for partially damped systems by spectral compensation. 2006. hal-00129724

\section{HAL Id: hal-00129724 \\ https://hal.science/hal-00129724}

Preprint submitted on 8 Feb 2007

HAL is a multi-disciplinary open access archive for the deposit and dissemination of scientific research documents, whether they are published or not. The documents may come from teaching and research institutions in France or abroad, or from public or private research centers.
L'archive ouverte pluridisciplinaire HAL, est destinée au dépôt et à la diffusion de documents scientifiques de niveau recherche, publiés ou non, émanant des établissements d'enseignement et de recherche français ou étrangers, des laboratoires publics ou privés. 


\section{Optimal energy decay rate for partially damped systems by spectral compensation}

\section{Paola LORETI * Bopeng RAO ${ }^{\dagger}$}

Abstract. We study the stability of weakly coupled and partially damped systems by means of Riesz basis approach in higher dimension spaces. We propose a weaker distributed damping that compensates the behaviour of the eigenvalues of the system, therefore gives the optimal polynomial energy decay rate for smooth initial data.

Key words. weaker damping, spectral compensation, optimal energy decay rate, Riesz basis in higher dimension space.

\section{AMS subject classifications 93D20}

$\S 1$. Introduction. The aim of this paper, the results of which were announced in [16], is to investigate the energy decay rate of the following weakly coupled and partially damped system

$$
\left\{\begin{array}{l}
y_{t t}+A y+B y_{t}+a u=0 \\
u_{t t}+A u+a y=0
\end{array}\right.
$$

where $a$ is real number, $A$ is a self-adjoint coercive operator and $B$ a linear bounded positive operator in a separated Hilbert space $H$. Assume furthermore that the resolvent of $A$ is compact in $H$. Then there exists an increasing sequence $\mu_{n}^{2} \rightarrow+\infty$ and an orthonormal sequence $e_{n} \in H$ such that

$$
A e_{n}=\mu_{n}^{2} e_{n}, \quad \forall n \geq 1
$$

Write (1.1) as

$$
\frac{d}{d t}\left(\begin{array}{l}
y \\
z \\
u \\
v
\end{array}\right)=\left(\begin{array}{c}
z \\
-A y-B z-a u \\
v \\
-A u-a y
\end{array}\right)=: \mathcal{A}\left(\begin{array}{l}
y \\
z \\
u \\
v
\end{array}\right) .
$$

* Dipartimento di Metodi e Modelli Matematici per le Scienze Applicate, Università degli Studi di Roma "La Sapienza", Via A. Scarpa n. 16, 00161 Roma, Italy.

$\ddagger$ Institut de Recherche Mathématique Avancée, Université Louis Pasteur de Strasbourg, 7 Rue René-Descartes, 67084 Strasbourg, France 
If $a$ is small enough, it was shown in [4] that the equation (1.3) generates a $C_{0}$-semigroup of contractions on the Hilbert space

$$
\mathcal{H}=D\left(A^{\frac{1}{2}}\right) \times H \times D\left(A^{\frac{1}{2}}\right) \times H .
$$

Moreover, let

$$
w_{n}=\frac{1}{\sqrt{2}}\left(\begin{array}{c}
0 \\
0 \\
\frac{e_{n}}{i \mu_{n}} \\
e_{n}
\end{array}\right) .
$$

Then using (1.2) a straightforward computation gives

$$
\left\|w_{n}\right\|_{\mathcal{H}}=1, \quad\left\|\left(i \mu_{n}-\mathcal{A}\right) w_{n}\right\|_{\mathcal{H}}^{2}=\frac{a^{2}}{2 \mu_{n}^{2}} \rightarrow 0 .
$$

This shows that the resolvent of $\mathcal{A}$ is not uniformly bounded on the imaginary axis. Following [7] and [18] (see also [15] for applications) the system (1.3) is not uniformly stable in $\mathcal{H}$.

Now assume that, and this is true in the case $B=I$, the spectrum of $\mathcal{A}$ has asymptotic expansions

$$
\lambda_{1, n}^{ \pm} \sim i \pm \mu_{n}-\frac{1}{2}, \quad \lambda_{2, n}^{ \pm} \sim i \pm \mu_{n}-\frac{a^{2}}{2 \mu_{n}^{2}} .
$$

Then the energy corresponding to the first branch of eigenvalues decays exponentially and that one corresponding to the second branch of eigenvalues decays only at the rate $1 / t$. Therefore the total energy decays at the rate $1 / t$.

Inspired by this remark, we look for a weaker damping operator for example $B=A^{\gamma}$ with $\gamma<0$. In that case, we have the following asymptotic expansions of the eigenvalues

$$
\left\{\begin{array}{lll}
\lambda_{1, n}^{ \pm} \sim \pm i \mu_{n}-\frac{\mu_{n}^{2 \gamma}}{2}, & \lambda_{2, n}^{ \pm} \sim \pm i \mu_{n}-\frac{a^{2}}{2 \mu_{n}^{2 \gamma+2}}, & 2 \gamma+1>0 \\
\lambda_{1, n}^{ \pm} \sim \pm i \mu_{n}-\frac{\mu_{n}^{2 \gamma}}{2}, & \lambda_{2, n}^{ \pm} \sim \pm i \mu_{n}-\frac{\mu_{n}^{2 \gamma}}{2}, & 2 \gamma+1<0
\end{array}\right.
$$

and the eigenvectors

$$
\begin{array}{ll}
e_{1, n}^{ \pm} \sim \frac{1}{\sqrt{2}}\left(\begin{array}{c}
\frac{e_{n}}{ \pm i \mu_{n}} \\
e_{n} \\
0 \\
0
\end{array}\right), \quad e_{2, n}^{ \pm} \sim \frac{1}{\sqrt{2}}\left(\begin{array}{c}
0 \\
0 \\
\frac{e_{n}}{ \pm i \mu_{n}} \\
e_{n}
\end{array}\right) \quad 2 \gamma+1>0, \\
e_{1, n}^{ \pm} \sim \frac{1}{2}\left(\begin{array}{c}
\frac{e_{n}}{ \pm i \mu_{n}} \\
\frac{e_{n}}{ \pm i \mu_{n}} \\
e_{n}
\end{array}\right), & e_{2, n}^{ \pm} \sim \frac{1}{2}\left(\begin{array}{c}
\frac{e_{n}}{\mp i \mu_{n}} \\
-e_{n} \\
\frac{e_{n}}{ \pm i \mu_{n}} \\
e_{n}
\end{array}\right) \quad 2 \gamma+1<0 .
\end{array}
$$


If $2 \gamma+1>0$, then the real part of $\lambda_{1, n}^{ \pm}$is of order $\mu_{n}^{2 \gamma}$ and $\lambda_{2, n}^{ \pm}$is of order $\frac{1}{\mu_{n}^{2 \gamma+2}}$. Therefore the energy corresponding to the first branch decays at the rate $t^{\frac{1}{\gamma}}$, and that one corresponding to the second branch decays at the rate $t^{-\frac{1}{\gamma+1}}$. Since $-\frac{1}{\gamma+1}>\frac{1}{\gamma}$, the total energy decays only at the rate $t^{-\frac{1}{\gamma+1}}$. In this case the eigenvectors $e_{1, n}^{ \pm}, e_{2, n}^{ \pm}$are asymptotically decoupled, and the two equations of (1.1) are very weakly coupled or almost independent.

If $2 \gamma+1<0$, then the real parts of $\lambda_{1, n}^{ \pm}$and $\lambda_{2, n}^{ \pm}$are of the same order $\mu_{n}^{2 \gamma}$. The total energy decays at the rate $t^{\frac{1}{\gamma}}$ and achieves the maximum decay $1 / t^{2}$ for $2 \gamma+1=0$. In that case the eigenvectors $e_{1, n}^{ \pm}$are well involved with the eigenvectors $e_{2, n}^{ \pm}$, and the two equations are really coupled.

The paper is organized as follows. In section 2, we establish a general result on polynomial decay rate of energy by a spectral approach. Section 3 was devoted to the study of the optimal decay rate of system (1.1) with a weaker damping $B=A^{\gamma}$ for $\gamma<0$. Under a suitable framework, we can establish the asymptotic expansions for the eigenvalues and the eigenvectors as in (1.6)(1.7). But these expansions couldn't give, except in one-dimension, the desired quadratical convergence for the usual Riesz basis approach. We will construct a sequence of pairwise orthogonal subspaces. By this way, we reduced the quadratical convergence to the Parseval equality. In order to obtain a Riesz basis we only need some weaker asymptotic expansions on the subspaces. In section 4, we give some examples of application.

There are many results concerning the polynomial decay rate. The majority was obtained by spectral approaches ([9], [8], [23], [24]) or frequency domain method ([5], [13], [14]). Others results were obtained by multiplier method ([4], [20], [21]). Also we mention [22] for a general formulation for partially damped systems and [12] for exact controllability and observability for coupled distributed systems.

$\S 2$. Optimal polynomial energy decay rate by spectral approach. We give a spectral approach for the polynomial energy decay rate of $C_{0}$-semigroups.

Theorem 2.1. Let $S(t)$ be a $C_{0}$ semigroup of contractions generated by the operator $\mathcal{A}$ on a Hilbert space $\mathcal{H}$. Let $\lambda_{k, n}(1 \leq k \leq K)$ denote the $k$-th branch of eigenvalues of $\mathcal{A}$ and $\left\{e_{k, n}\right\}_{1 \leq k \leq K, n \geq 1}$ the system of eigenvectors which forms a Riesz basis in $\mathcal{H}$. Assume that for each $1 \leq k \leq K$ there exist a positive sequence $\mu_{k, n} \rightarrow+\infty$ as $n \rightarrow+\infty$ and two positive constants $\alpha_{k} \geq 0, \beta_{k}>0$ such that

$$
\operatorname{Re} \lambda_{k, n} \leq-\frac{\beta_{k}}{\mu_{k, n}^{\alpha_{k}}} \quad \text { and } \quad\left|\operatorname{Im} \lambda_{k, n}\right| \geq \mu_{k, n}, \quad \forall n \geq 1
$$


Then for any $u_{0} \in D\left(\mathcal{A}^{\theta}\right)$ with $\theta>0$, there exists a constant $M>0$ independent of $u_{0}$ such that

$$
\left\|S(t) u_{0}\right\|_{\mathcal{H}}^{2} \leq\left\|\mathcal{A}^{\theta} u_{0}\right\|_{\mathcal{H}}^{2} \frac{M}{t^{2 \theta \delta}}, \quad \forall t>0
$$

where the decay rate $\delta$ is given by

$$
\delta:=\min _{1 \leq k \leq K} \frac{1}{\alpha_{k}}=\frac{1}{\alpha_{l}} .
$$

Moreover if there exists a constant $c_{1}>0, c_{2}>0$ such that

$$
\operatorname{Re} \lambda_{l, n} \geq-\frac{c_{1}}{\mu_{l, n}^{\alpha_{l}}} \quad \text { and } \quad\left|\operatorname{Im} \lambda_{l, n}\right| \leq c_{2} \mu_{l, n} \quad \forall n \geq 1
$$

then the decay rate $\delta$ given in (2.3) is optimal.

Proof. Since $\left\{e_{k, n}\right\}_{1 \leq k \leq K, n \geq 1}$ is a Riesz basis in $\mathcal{H}$, any $u_{0} \in D\left(\mathcal{A}^{\theta}\right)$ can be written as

$$
u_{0}=\sum_{k=1}^{K} \sum_{n=1}^{+\infty} a_{k, n} e_{k, n}
$$

Moreover there exist two constants $C_{1}>0, C_{2}>0$ such that

$$
C_{1} \sum_{k=1}^{K} \sum_{n=1}^{+\infty}\left|a_{k, n}\right|^{2} \leq\left\|u_{0}\right\|_{\mathcal{H}}^{2} \leq C_{2} \sum_{k=1}^{K} \sum_{n=1}^{+\infty}\left|a_{k, n}\right|^{2}
$$

Using the expansion (2.5) and the continuity of the semigroup $S(t)$, we get

$$
S(t) u_{0}=\sum_{k=1}^{K} \sum_{n=1}^{+\infty} a_{k, n} e^{\lambda_{k, n} t} e_{k, n}
$$

Then using the first conditions of (2.1) and the second inequality of (2.6) into (2.7), we obtain

$$
\left\|S(t) u_{0}\right\|_{\mathcal{H}}^{2} \leq \sup _{1 \leq k \leq K} \sup _{n \geq 1}\left(\frac{C_{2}}{\mu_{k, n}^{2 \theta} \exp \left(\frac{2 \beta_{k} t}{\mu_{k, n}^{\alpha}}\right)}\right) \sum_{k=1}^{K} \sum_{n=1}^{+\infty}\left|a_{k, n}\right|^{2} \mu_{k, n}^{2 \theta} .
$$

On the other hand, the conditions of (2.1) imply that there exists a positive constant $C_{3}>0$ such that

$$
\sum_{k=1}^{K} \sum_{n=1}^{+\infty} \mu_{k, n}^{2 \theta}\left|a_{k, n}\right|^{2} \leq C_{3} \sum_{k=1}^{K} \sum_{n=1}^{+\infty}\left|\lambda_{k, n}\right|^{2 \theta}\left|a_{k, n}\right|^{2} \leq \frac{C_{3}}{C_{1}}\left\|\mathcal{A}^{\theta} u_{0}\right\|_{\mathcal{H}}^{2}
$$


Then inserting (2.9) into (2.8), we get

$$
\left\|S(t) u_{0}\right\|_{\mathcal{H}}^{2} \leq \sup _{1 \leq k \leq K} \sup _{n \geq 1}\left(\frac{C_{2} C_{3}}{C_{1} \mu_{k, n}^{2 \theta} \exp \left(\frac{2 \beta_{k} t}{\mu_{k, n}^{\alpha}}\right)}\right)\left\|\mathcal{A}^{\theta} u_{0}\right\|_{\mathcal{H}}^{2}
$$

If $\alpha_{k}=0$ for some $1 \leq k \leq K$, since $\inf _{n \geq 1} \mu_{k, n}>0$, then it is easy to find a constant $M_{k}>0$ such that

$$
\frac{1}{\mu_{k, n}^{2 \theta} \exp \left(2 \beta_{k} t\right)} \leq \frac{M_{k}}{t^{2 \theta \delta}}, \quad \forall n \geq 1 .
$$

If $\alpha_{k}>0$ for some $1 \leq k \leq K$, then putting

$$
\frac{t}{\mu_{k, n}^{\alpha_{k}}}=\frac{1}{x}, \quad f_{k}(x)=x^{\frac{2 \theta}{\alpha_{k}}} e^{\frac{2 \beta_{k}}{x}}
$$

we rewrite

$$
\mu_{k, n}^{2 \theta} \exp \left(\frac{2 \beta_{k} t}{\mu_{k, n}^{\alpha_{k}}}\right)=f_{k}(x) t^{\frac{2 \theta}{\alpha_{k}}}
$$

Then a straightforward computation gives

$$
f_{k}^{\prime}(x)=2 e^{\frac{\beta_{k}}{x}} x^{\frac{2 \theta}{\alpha_{k}}-2}\left(\frac{\theta}{\alpha_{k}} x-\beta_{k}\right) \quad \begin{cases}>0, & x>\frac{\alpha_{k} \beta_{k}}{\theta} \\ <0, & x<\frac{\alpha_{k} \beta_{k}}{\theta} .\end{cases}
$$

It follows that

$$
\inf _{x>0} f_{k}(x)=f_{k}\left(\frac{\alpha_{k} \beta_{k}}{\theta}\right)=\left(\frac{\alpha_{k} \beta_{k}}{\theta}\right)^{\frac{2 \theta}{\alpha_{k}}} e^{\frac{2 \theta}{\alpha_{k}}}:=M_{k}^{-1}>0 .
$$

Inserting (2.13) into (2.12) gives that

$$
\frac{1}{\mu_{k, n}^{2 \theta} \exp \left(\frac{2 \beta_{k} t}{\mu_{k, n}^{\alpha}}\right)} \leq \frac{M_{k}}{t^{\frac{2 \theta}{\alpha_{k}}}}, \quad n \geq 1
$$

Finally combining the cases (2.11) and (2.14), we get the polynomial energy decay rate $(2.2)$ with the constant $M$ given by

$$
M=\frac{C_{2} C_{3}}{C_{1}} \max _{1 \leq k \leq K} M_{k}
$$

Now we consider the optimality of $\delta$. To simplify the notations, we write

$$
\alpha_{l}=\alpha, \quad \mu_{l, n}=\mu_{n}, \quad \lambda_{l, n}=\lambda_{n}, \quad e_{l, n}=e_{n} .
$$


Since $\mu_{n} \rightarrow+\infty$, then for any $\epsilon>0$, we can find a subsequence, still denoted by $\mu_{n}$, such that

$$
\sum_{n=1}^{\infty} \frac{1}{\mu_{n}^{\alpha \epsilon}}<+\infty
$$

Then putting

$$
u_{0}=\sum_{n=1}^{\infty} \frac{C_{4}}{\mu_{n}^{(\theta+\alpha \epsilon / 2)}} e_{n}
$$

we see that $u_{0} \in D\left(\mathcal{A}^{\theta}\right)$ due to the convergence (2.16). On the other hand, thank to the second condition of (2.4), we can choose a constant $C_{4}>0$ such that $\left\|\mathcal{A}^{\theta} u_{0}\right\|_{\mathcal{H}}=1$. Now using the first condition of $(2.4)$, we get

$$
\left\|S(t) u_{0}\right\|_{\mathcal{H}}^{2}=\left\|\sum_{n=1}^{\infty} \frac{C_{4} e^{\lambda_{n} t} e_{n}}{\mu_{n}^{(\theta+\alpha \epsilon / 2)}}\right\|_{\mathcal{H}}^{2} \geq \frac{C_{1} C_{4}^{2}}{\mu_{m}^{2 \theta+\alpha \epsilon} \exp \left(\frac{2 c_{1} t}{\mu_{m}^{\alpha}}\right)}, \quad \forall m \geq 1
$$

Finally setting $t_{m}=\mu_{m}^{\alpha}$ in (2.18), we obtain that

$$
\left\|S\left(t_{m}\right) u_{0}\right\|_{\mathcal{H}}^{2} \geq \frac{C_{1} C_{4}^{2}}{e^{2 c_{1}} \mu_{m}^{2 \theta+\alpha \epsilon}}=\frac{C_{1} C_{4}^{2}}{e^{2 c_{1}} t_{m}^{2 \theta \delta+\epsilon}}, \quad \forall m \geq 1
$$

This means that the trajectory $S(t) u_{0}$ decays slower than $\frac{1}{t^{2 \theta \delta+\epsilon}}$ on the time sequence $t_{m} \rightarrow+\infty$. Then for any $\epsilon>0$, we can't expect the decay rate $\frac{1}{t^{2 \theta \delta+\epsilon}}$ for all initial data $u_{0} \in D\left(\mathcal{A}^{\theta}\right)$ and for all $t>0$. The proof is thus complete.

Remark 2.1. If $\lambda_{k, n}$ is an eigenvalue of algebraic multiplicity $d>1$, then the corresponding factor in (2.8) and (2.14) will be replaced by

$$
\frac{|p(t)|^{2}}{\mu_{k, n}^{2 \theta} \exp \left(\frac{2 \beta_{k} t}{\mu_{k, n}^{\alpha}}\right)} \leq \frac{M_{k}}{t^{2 \theta \delta}}
$$

where $p(t)$ is a polynomial of degree $d-1$ and $M_{k}>0$ is a constant. Therefore, Theorem 2.1 remains valid if the operator $\mathcal{A}$ admits a finite number of algebraically multiple eigenvalues and the system of root vectors forms a Riesz basis in $\mathcal{H}$.

Remark 2.2. Theorem 2.1 is valid for all initial data $u_{0} \in D\left(\mathcal{A}^{\theta}\right)$ with $\theta>0$. This is different from an earlier result of Littman-Markus in [11] where the initial data $u_{0}$ should satisfy some stronger conditions. 
$\S 3$. Spectral compensation for weakly damped systems. Let $A$ be a densely defined closed self-adjoint operator in a Hilbert space $H$ such that for some positive constant $c$ we have

$$
(A u, u) \geq c\|u\|_{H}^{2}, \quad \forall u \in D(A) .
$$

Let $\gamma \leq 0$, and $a$ be a small real number. We consider the following weakly coupled equations

$$
\begin{aligned}
& y_{t t}+A y+A^{\gamma} y_{t}+a u=0, \\
& u_{t t}+A u+a y=0 .
\end{aligned}
$$

In order to curry the explicit computation of the eigenvalues, we have chosen the same operator $A$ in the two equations of the system (3.2)-(3.3). The results of this section complete and improve a recent work of Alabau-Cannarsa-Komornik [4].

Define the Hilbert space

$$
\mathcal{H}=D\left(A^{\frac{1}{2}}\right) \times H \times D\left(A^{\frac{1}{2}}\right) \times H
$$

equipped with the equivalent inner product

$$
\begin{aligned}
\langle(y, z, u, v),(f, g, p, q)\rangle_{\mathcal{H}}= & \left(A^{\frac{1}{2}} y, A^{\frac{1}{2}} f\right)+(z, g)+\left(A^{\frac{1}{2}} u, A^{\frac{1}{2}} p\right)+(v, q) \\
& +a((y, p)+(u, f)) .
\end{aligned}
$$

Setting

$$
\begin{aligned}
& D(\mathcal{A})=\{w=\left.(y, z, u, v)^{T} \in \mathcal{H} \quad z, v \in D\left(A^{\frac{1}{2}}\right), \quad y, u \in D(A)\right\}, \\
& \mathcal{A}\left(\begin{array}{l}
y \\
z \\
u \\
v
\end{array}\right)=\left(\begin{array}{c}
z \\
-A y-A^{\gamma} z-a u \\
v \\
-A u-a y
\end{array}\right)
\end{aligned}
$$

we can write the system (3.2)-(3.3) into an evolution equation

$$
\frac{d}{d t} w=\mathcal{A} w, \quad w(0)=w_{0} .
$$

Under the condition (3.1), we can prove easily that $\mathcal{A}$ is a maximal dissipative operator on $\mathcal{H}$ provided that $a$ is small enough. Therefore $\mathcal{A}$ generates a $C^{0}$ semigroup of contractions on $\mathcal{H}$ (Theorem 1.4.3 in [17]). Moreover, setting the energy by

$$
E(t)=\frac{1}{2}\left(\left\|A^{\frac{1}{2}} y\right\|^{2}+\left\|y_{t}\right\|^{2}+\left\|A^{\frac{1}{2}} u\right\|^{2}+\left\|u_{t}\right\|^{2}+a(y, u)+a(u, y)\right)
$$


then a straightforward computation gives that

$$
\frac{d}{d t} E(t)=-\left\|A^{\gamma} y_{t}\right\|^{2} \leq 0
$$

Assume furthermore that the resolvent of $A$ is compact in $H$. Then there exists an increasing sequence $\mu_{n}^{2} \rightarrow+\infty$ and a orthonormal sequence $e_{n} \in H$ such that

$$
A e_{n}=\mu_{n}^{2} e_{n}, \quad \forall n \geq 1
$$

Moreover the system $\left\{e_{n}\right\}_{n \geq 1}$ forms a Hilbert basis on $H$.

Now let $\lambda$ be an eigenvalue and $(y, z, u, v)$ be the associated eigenvector of the operator $\mathcal{A}$. Then we have

$$
\left\{\begin{array}{l}
z=\lambda y \\
-A y-A^{\gamma} z-a u=\lambda z \\
v=\lambda u \\
-A u-a y=\lambda v
\end{array}\right.
$$

We will see in Proposition 3.3 that all eigenvectors of $\mathcal{A}$ are of the following form

$$
y=\alpha_{n} e_{n}, \quad z=\lambda \alpha_{n} e_{n}, \quad u=\beta_{n} e_{n}, \quad v=\lambda \beta_{n} e_{n}
$$

Inserting (3.7) into (3.6) we get

$$
\left\{\begin{array}{l}
\left(\lambda^{2}+\mu_{n}^{2}+\lambda \mu_{n}^{2 \gamma}\right) \alpha_{n}+a \beta_{n}=0 \\
a \alpha_{n}+\left(\lambda^{2}+\mu_{n}^{2}\right) \beta_{n}=0
\end{array}\right.
$$

which has non trivial solution $\left(\alpha_{n}, \beta_{n}\right) \neq(0,0)$ if and only if $\lambda$ is a solution of the equation

$$
\left(\lambda^{2}+\lambda \mu_{n}^{2 \gamma}+\mu_{n}^{2}\right)\left(\lambda^{2}+\mu_{n}^{2}\right)=a^{2}
$$

Proposition 3.1. For each $\mu_{n}>0$, the corresponding eigenvalues $\lambda_{1, n}^{ \pm}, \lambda_{2, n}^{ \pm}$ of the system (3.2)-(3.3) satisfy the following asymptotic expansions.

I. If $-1 / 2<\gamma \leq 0$,

$$
\begin{aligned}
& \lambda_{1, n}^{ \pm}= \pm i \mu_{n}-\frac{\mu_{n}^{2 \gamma}}{2}+O\left(\frac{1}{\mu_{n}}\right) \\
& \lambda_{2, n}^{ \pm}= \pm i \mu_{n}-\frac{a^{2}}{2 \mu_{n}^{2 \gamma+2}}+O\left(\frac{1}{\mu_{n}^{6 \gamma+4}}\right)
\end{aligned}
$$


II. If $\gamma<-1 / 2$,

$$
\begin{aligned}
& \lambda_{1, n}^{ \pm}= \pm i \sqrt{\mu_{n}^{2}+a}-\frac{\mu_{n}^{2 \gamma}}{2}+O\left(\mu_{n}^{4 \gamma+1}\right), \\
& \lambda_{2, n}^{ \pm}= \pm i \sqrt{\mu_{n}^{2}-a}-\frac{\mu_{n}^{2 \gamma}}{2}+O\left(\mu_{n}^{4 \gamma+1}\right) .
\end{aligned}
$$

III. If $\gamma=-1 / 2$,

$$
\begin{aligned}
& \lambda_{1, n}^{ \pm}= \pm i \mu_{n}-\frac{1 \pm \sqrt{1-4 a^{2}}}{4 \mu_{n}}+O\left(\frac{1}{\mu_{n}^{2}}\right), \\
& \lambda_{2, n}^{ \pm}= \pm i \mu_{n}-\frac{1 \mp \sqrt{1-4 a^{2}}}{4 \mu_{n}}+O\left(\frac{1}{\mu_{n}^{2}}\right) .
\end{aligned}
$$

Proof. Firstly, let $\lambda_{n}$ be one of the four solutions of the equation (3.9). If $\left|\lambda_{n}^{2}+\mu_{n}^{2}\right| \leq 1$, then it follows that

$$
\frac{\lambda_{n}}{\mu_{n}}= \pm i+O\left(\mu_{n}^{-2}\right) .
$$

If $\left|\lambda_{n}^{2}+\mu_{n}^{2}\right| \geq 1$, then from the equation (3.9) we get

$$
\left(\frac{\lambda_{n}}{\mu_{n}}\right)^{2}+\frac{\lambda_{n}}{\mu_{n}} \mu_{n}^{2 \gamma-1}+1=O\left(\mu_{n}^{-2}\right) .
$$

It follows that

$$
\frac{\lambda_{n}}{\mu_{n}}= \pm i+O\left(\mu_{n}^{2 \gamma-1}\right) .
$$

Combining (3.16) and (3.17), we get

$$
\frac{\lambda_{n}}{\mu_{n}}= \pm i+O\left(\mu_{n}^{2 \gamma-1}\right)+O\left(\mu_{n}^{-2}\right) .
$$

Next, solving the equation (3.9), we get

$$
2\left(\lambda^{2}+\mu_{n}^{2}\right)=-\lambda \mu_{n}^{2 \gamma} \mp \sqrt{\lambda^{2} \mu_{n}^{4 \gamma}+4 a^{2}} .
$$

The symbol $a \sim b$ means that $a-b \rightarrow 0$. In the following computations we use many times the expansion $\sqrt{1+z}=1+z / 2+O\left(z^{2}\right)$. 
Case I : $-1 / 2<\gamma \leq 0$. From (3.18), we have

$$
\left|\lambda_{n} \mu_{n}^{2 \gamma}\right| \sim \mu_{n}^{1+2 \gamma} \rightarrow+\infty .
$$

Using the asymptotic expansion (3.20) into (3.19), we get

$$
\left\{\begin{array}{l}
\lambda_{1, n}^{2}=-\mu_{n}^{2}-\lambda_{1, n} \mu_{n}^{2 \gamma}+O\left(\frac{1}{\mu_{n}^{2 \gamma+1}}\right), \\
\lambda_{2, n}^{2}=-\mu_{n}^{2}+\frac{a^{2}}{\lambda_{2, n} \mu_{n}^{2 \gamma}}+O\left(\frac{1}{\mu_{n}^{6 \gamma+3}}\right) .
\end{array}\right.
$$

It follows from (3.21) that

$$
\left\{\begin{array}{l}
\lambda_{1, n}^{ \pm}= \pm i \mu_{n} \pm \frac{i \lambda_{1, n} \mu_{n}^{2 \gamma-1}}{2}+O\left(\frac{1}{\mu_{n}}\right), \\
\lambda_{2, n}^{ \pm}= \pm i \mu_{n} \mp \frac{i a^{2}}{2 \lambda_{2, n} \mu_{n}^{2 \gamma+1}}+O\left(\frac{1}{\mu_{n}^{6 \gamma+4}}\right) .
\end{array}\right.
$$

In particular, we get

$$
\left\{\begin{array}{l}
\lambda_{1, n}^{ \pm}= \pm i \mu_{n}+O\left(\mu_{n}^{2 \gamma}\right), \\
\lambda_{2, n}^{ \pm}= \pm i \mu_{n}+O\left(\frac{1}{\mu_{n}^{2 \gamma+2}}\right) .
\end{array}\right.
$$

Inserting (3.23) into (3.22) gives that

$$
\begin{aligned}
& \lambda_{1, n}^{ \pm}= \pm i \mu_{n}-\frac{\mu_{n}^{2 \gamma}}{2}+O\left(\frac{1}{\mu_{n}}\right) \\
& \lambda_{2, n}^{ \pm}= \pm i \mu_{n}-\frac{a^{2}}{2 \mu_{n}^{2 \gamma+2}}+O\left(\frac{1}{\mu_{n}^{6 \gamma+4}}\right) .
\end{aligned}
$$

Case II $: \gamma<-1 / 2$. From (3.18) we have

$$
\left|\lambda_{n} \mu_{n}^{2 \gamma}\right| \sim\left|\mu_{n}^{1+2 \gamma}\right| \rightarrow 0 .
$$

Using the asymptotic expansion (3.26) into (3.19), we have

$$
\left\{\begin{array}{l}
\lambda_{1, n}^{2}=-\left(\mu_{n}^{2}+a\right)-\frac{\lambda_{1, n} \mu_{n}^{2 \gamma}}{2}+O\left(\mu_{n}^{4 \gamma+2}\right), \\
\lambda_{2, n}^{2}=-\left(\mu_{n}^{2}-a\right)-\frac{\lambda_{1, n} \mu_{n}^{2 \gamma}}{2}+O\left(\mu_{n}^{4 \gamma+2}\right) .
\end{array}\right.
$$


It follows from (3.27)

$$
\left\{\begin{array}{l}
\lambda_{1, n}^{ \pm}= \pm i \sqrt{\mu_{n}^{2}+a} \pm \frac{i \lambda_{1, n} \mu_{n}^{2 \gamma}}{2 \sqrt{\mu_{n}^{2}+a}}+O\left(\mu_{n}^{4 \gamma+1}\right) \\
\lambda_{2, n}^{ \pm}= \pm i \sqrt{\mu_{n}^{2}-a} \pm \frac{i \lambda_{1, n} \mu_{n}^{2 \gamma}}{2 \sqrt{\mu_{n}^{2}-a}}+O\left(\mu_{n}^{4 \gamma+1}\right)
\end{array}\right.
$$

In particular, we get

$$
\left\{\begin{array}{l}
\lambda_{1, n}^{ \pm}= \pm i \sqrt{\mu_{n}^{2}+a}+O\left(\mu_{n}^{2 \gamma}\right) \\
\lambda_{2, n}^{ \pm}= \pm i \sqrt{\mu_{n}^{2}-a}+O\left(\mu_{n}^{2 \gamma}\right)
\end{array}\right.
$$

Inserting (3.29) into (3.28) we have

$$
\left\{\begin{array}{l}
\lambda_{1, n}^{ \pm}= \pm i \sqrt{\mu_{n}^{2}+a}-\frac{\mu_{n}^{2 \gamma}}{2}+O\left(\mu_{n}^{4 \gamma+1}\right), \\
\lambda_{2, n}^{ \pm}= \pm i \sqrt{\mu_{n}^{2}-a}-\frac{\mu_{n}^{2 \gamma}}{2}+O\left(\mu_{n}^{4 \gamma+1}\right) .
\end{array}\right.
$$

Case III : $\gamma=-1 / 2$. From (3.18) we have

$$
\frac{\lambda_{1, n}^{ \pm}}{\mu_{n}} \sim \pm i+O\left(\frac{1}{\mu_{n}^{2}}\right), \quad \frac{\lambda_{2, n}^{ \pm}}{\mu_{n}} \sim \pm i+O\left(\frac{1}{\mu_{n}^{2}}\right) .
$$

Using the asymptotic expansion (3.31) into (3.19), we have

$$
\left\{\begin{array}{l}
\lambda_{1, n}^{2}=-\mu_{n}^{2} \mp \frac{i}{2}\left(1 \pm \sqrt{1-4 a^{2}}\right)+O\left(\frac{1}{\mu_{n}^{2}}\right), \\
\lambda_{2, n}^{2}=-\mu_{n}^{2} \mp \frac{i}{2}\left(1 \mp \sqrt{1-4 a^{2}}\right)+O\left(\frac{1}{\mu_{n}^{2}}\right) .
\end{array}\right.
$$

It follows from (3.32) that

$$
\begin{aligned}
& \lambda_{1, n}^{ \pm}= \pm i \mu_{n}-\frac{1 \pm \sqrt{1-4 a^{2}}}{4 \mu_{n}}+O\left(\frac{1}{\mu_{n}^{3}}\right), \\
& \lambda_{2, n}^{ \pm}= \pm i \mu_{n}-\frac{1 \mp \sqrt{1-4 a^{2}}}{4 \mu_{n}}+O\left(\frac{1}{\mu_{n}^{3}}\right) .
\end{aligned}
$$

The proof is thus complete. 
If $\lambda_{1, n}^{ \pm}, \lambda_{2, n}^{ \pm}$are simple eigenvalues, then setting

$$
\begin{aligned}
& \beta_{1, n}^{ \pm}=-\frac{a}{\left(\lambda_{1, n}^{ \pm}\right)^{2}+\mu_{n}^{2}} \alpha_{1, n}^{ \pm}, \\
& \alpha_{2, n}^{ \pm}=-\frac{\left(\lambda_{2, n}^{ \pm}\right)^{2}+\mu_{n}^{2}}{a} \beta_{2, n}^{ \pm}
\end{aligned}
$$

in $(3.8)$ we get

$$
e_{1, n}^{ \pm}=\alpha_{1, n}^{ \pm}\left(\begin{array}{c}
\frac{e_{n}}{\lambda_{1, n}^{ \pm}} \\
e_{n} \\
-\frac{a e_{n}}{\lambda_{1, n}^{ \pm}\left(\left(\lambda_{1, n}^{ \pm}\right)^{2}+\mu_{n}^{2}\right)} \\
-\frac{a e_{n}}{\left(\lambda_{1, n}^{ \pm}\right)^{2}+\mu_{n}^{2}}
\end{array}\right), \quad e_{2, n}^{ \pm}=\beta_{2, n}^{ \pm}\left(\begin{array}{c}
-\frac{\left(\left(\lambda_{2, n}^{ \pm}\right)^{2}+\mu_{n}^{2}\right) e_{n}}{a \lambda_{2, n}^{ \pm}} \\
-\frac{\left(\left(\lambda_{2, n}^{ \pm}\right)^{2}+\mu_{n}^{2}\right) e_{n}}{a} \\
\frac{e_{n}}{\lambda_{2, n}^{ \pm}} \\
e_{n}
\end{array}\right)
$$

Now let us denote by $V_{n}$ the eigen-space corresponding to the four eigenvalues $\lambda_{1, n}^{ \pm}, \lambda_{2, n}^{ \pm}$:

$$
V_{n}=\operatorname{Sp}\left\{e_{1, n}^{+}, e_{2, n}^{+}, e_{1, n}^{-}, e_{2, n}^{-}\right\} .
$$

From the expressions in (3.37), the subspaces $\left\{V_{n}\right\}_{n \geq 1}$ are clearly pairwise orthogonal in $\mathcal{H}$. It is possible that the equation (3.9) has multiple solutions for some special parameters $\mu_{n}, a$. However for $a$ small enough, the Rouché theorem shows that the equation (3.9) admits at most a double solution for the first branch of eigenvalues $\lambda_{1, n}^{ \pm}=\lambda_{1, n}, e_{1, n}^{ \pm}=e_{1, n}$ which satisfies the following equation of derivation

$$
\left(2 \lambda+\mu_{n}^{2 \gamma}\right)\left(\lambda^{2}+\mu_{n}^{2}\right)+2 \lambda\left(\lambda^{2}+\lambda \mu_{n}^{2 \gamma}+\mu_{n}^{2}\right)=0
$$

In that case, we look for the corresponding root vector $\widetilde{e}_{1, n}=\left(\widetilde{y}_{n}, \widetilde{z}_{n}, \widetilde{u}_{n}, \widetilde{v}_{n}\right)$ such that

$$
\left(I-\lambda_{1, n} \mathcal{A}\right) \widetilde{e}_{1, n}=e_{1, n}
$$

which involves that

$$
\left\{\begin{array}{l}
\widetilde{z}_{n}-\lambda_{1, n} \widetilde{y}_{n}=\frac{e_{n}}{\lambda_{1, n}} \\
-A \widetilde{y}_{n}-A^{\gamma} \widetilde{z}_{n}-a \widetilde{u}_{n}-\lambda \widetilde{z}_{n}=e_{n} \\
\widetilde{v}_{n}-\lambda_{1, n} \widetilde{u}_{n}=-\frac{a e_{n}}{\lambda_{1, n}\left(\lambda_{1, n}^{2}+\mu_{n}^{2}\right)} \\
-A \widetilde{u}_{n}-a \widetilde{y}_{n}-\lambda \widetilde{v}_{n}=-\frac{a e_{n}}{\left(\lambda_{1, n}^{2}+\mu_{n}^{2}\right)}
\end{array}\right.
$$


Setting

$$
\widetilde{y}_{n}=\alpha_{1, n} e_{n}, \quad \widetilde{u}_{n}=\beta_{1, n} e_{n}
$$

in (3.39) we get

$$
\left\{\begin{array}{l}
\widetilde{z}_{n}=\alpha_{1, n} \lambda_{1, n} e_{n}+\frac{e_{n}}{\lambda_{1, n}}, \\
\widetilde{v}_{n}=\beta_{1, n} \lambda_{1, n} e_{n}-\frac{a e_{n}}{\lambda_{1, n}\left(\lambda_{1, n}^{2}+\mu_{n}^{2}\right)}
\end{array}\right.
$$

where the constants $\alpha_{1, n}$ and $\beta_{1, n}$ satisfy

$$
\left\{\begin{array}{l}
-\left(\mu^{2}+\lambda_{1, n} \mu^{2 \gamma}+\lambda_{1, n}^{2}\right) \alpha_{1, n}-a \beta_{1, n}=\frac{2 \lambda_{1, n}+\mu^{2 \gamma}}{\lambda_{1, n}} \\
-a \alpha_{1, n}-\left(\mu^{2}+\lambda_{1, n}^{2}\right) \beta_{1, n}=-\frac{2 a}{\lambda_{1, n}^{2}+\mu_{n}^{2}}
\end{array}\right.
$$

Since $\lambda_{1, n}$ satisfies (3.9) and (3.38), the first equation of (3.42) can be reduced to the second one. Therefore choosing

$$
\alpha_{1, n}=\frac{2}{\lambda_{1, n}^{2}+\mu_{n}^{2}}, \quad \beta_{1, n}=0 .
$$

in (3.40)-(3.41) we get the corresponding root vector

$$
\widetilde{e}_{1, n}=\left(\begin{array}{c}
\frac{2}{\lambda_{1, n}^{2}+\mu_{n}^{2}} e_{n} \\
\left(\frac{2 a \lambda_{1, n}}{\lambda_{1, n}^{2}+\mu_{n}^{2}}+\frac{1}{\lambda_{1, n}}\right) e_{n} \\
0 \\
-\frac{a}{\lambda_{1, n}\left(\lambda_{1, n}^{2}+\mu_{n}^{2}\right)} e_{n}
\end{array}\right) .
$$

Accordingly, we modify the subspace $V_{n}$ as

$$
V_{n}=\operatorname{Sp}\left\{e_{1, n}, e_{2, n}^{+}, \widetilde{e}_{1, n}, e_{2, n}^{-}\right\} .
$$

Once again, the expression (3.44) shows that the subspaces $\left\{V_{n}\right\}_{n \geq 1}$ are still pairwise orthogonal in $\mathcal{H}$.

Proposition 3.2. Let $E_{1, n}^{ \pm}, E_{2, n}^{ \pm}$be the eigenvectors of the decoupled system (corresponding to $a=0$ )

$$
E_{1, n}^{ \pm}=\frac{1}{\sqrt{2}}\left(\begin{array}{c}
\frac{e_{n}}{ \pm i \mu_{n}} \\
e_{n} \\
0 \\
0
\end{array}\right), \quad E_{2, n}^{ \pm}=\frac{1}{\sqrt{2}}\left(\begin{array}{c}
0 \\
0 \\
\frac{e_{n}}{ \pm i \mu_{n}} \\
e_{n}
\end{array}\right)
$$


Then the following relationship holds

$$
\left(e_{1, n}^{+}, e_{2, n}^{+}, e_{1, n}^{-}, e_{2, n}^{-}\right)=\left(E_{1, n}^{+}, E_{2, n}^{+}, E_{1, n}^{-}, E_{2, n}^{-}\right) L_{n}
$$

where $L_{n}$ is a $4 \times 4$ matrix such as

$$
\begin{aligned}
(3.47) L_{n} & =\left(\begin{array}{cccc}
1 & 0 & 0 & 0 \\
0 & 1 & 0 & 0 \\
0 & 0 & 1 & 0 \\
0 & 0 & 0 & 1
\end{array}\right)+O\left(\frac{1}{\mu_{n}^{2 \gamma+1}}\right) \quad 2 \gamma+1>0, \\
(3.48) L_{n} & =\frac{1}{\sqrt{2}}\left(\begin{array}{cccc}
1 & -1 & 0 & 0 \\
1 & 1 & 0 & 0 \\
0 & 0 & 1 & -1 \\
0 & 0 & 1 & 1
\end{array}\right)+O\left(\frac{1}{\mu_{n} \min \{2,-(2 \gamma+1)\}}\right) 2 \gamma+1<0 \\
(3.49) L_{n} & =\sqrt{2}\left(\begin{array}{cccc}
\sqrt{a}+ & \sqrt{a} & 0 & 0 \\
\frac{i a}{\sqrt{a}} & \frac{i a}{\sqrt{a}_{-}} & 0 & 0 \\
0 & 0 & \sqrt{a_{-}} & \sqrt{a_{+}} \\
0 & 0 & -\frac{i a}{\sqrt{a}_{-}} & -\frac{i a}{\sqrt{a}_{+}}
\end{array}\right)+O\left(\frac{1}{\mu_{n}^{2}}\right) \quad 2 \gamma+1=0
\end{aligned}
$$

where we have put

$$
a_{ \pm}=\frac{1 \pm \sqrt{1-4 a^{2}}}{2}
$$

Proof. For $2 \gamma+1>0$, using the asymptotic expansions (3.10)-(3.11), we have

$$
\begin{array}{ll}
\frac{1}{\left(\lambda_{1, n}^{ \pm}\right)^{2}+\mu_{n}^{2}}=O\left(\frac{1}{\mu_{n}^{2 \gamma+1}}\right), & \frac{1}{\lambda_{1, n}^{ \pm}}=\frac{1}{ \pm i \mu_{n}}+O\left(\mu_{n}^{2 \gamma-2}\right), \\
\left(\lambda_{2, n}^{ \pm}\right)^{2}+\mu_{n}^{2}=O\left(\frac{1}{\mu_{n}^{2 \gamma+1}}\right), & \frac{1}{\lambda_{2, n}^{ \pm}}=\frac{1}{ \pm i \mu_{n}}+O\left(\frac{1}{\mu_{n}^{2 \gamma+4}}\right) .
\end{array}
$$

Inserting (3.51)-(3.52) into (3.37) gives

$$
\begin{aligned}
& e_{1, n}^{ \pm}=\frac{1}{\sqrt{2}}\left(\begin{array}{c}
\frac{e_{n}}{ \pm i \mu_{n}} \\
e_{n} \\
0 \\
0
\end{array}\right)+O\left(\frac{1}{\mu_{n}^{2 \gamma+1}}\right), \\
& e_{2, n}^{ \pm}=\frac{1}{\sqrt{2}}\left(\begin{array}{c}
0 \\
0 \\
\frac{e_{n}}{ \pm i \mu_{n}} \\
e_{n}
\end{array}\right)+O\left(\frac{1}{\mu_{n}^{2 \gamma+1}}\right) .
\end{aligned}
$$


For $2 \gamma+1<0$, using (3.12)-(3.13), we have

$$
\begin{aligned}
& \frac{1}{\left(\lambda_{1, n}^{ \pm}\right)^{2}+\mu_{n}^{2}}=-\frac{1}{a}+O\left(\mu_{n}^{2 \gamma+1}\right), \quad \frac{1}{\lambda_{1, n}^{ \pm}}=\frac{1}{ \pm i \mu_{n}}+O\left(\frac{1}{\mu_{n}^{3}}\right) \\
& \frac{1}{\lambda_{2, n}^{ \pm}}=\frac{1}{ \pm i \mu_{n}}+O\left(\frac{1}{\mu_{n}^{3}}\right), \quad\left(\lambda_{2, n}^{ \pm}\right)^{2}+\mu_{n}^{2}=a+O\left(\mu_{n}^{2 \gamma+1}\right) .
\end{aligned}
$$

Inserting (3.55)-(3.56) into (3.37) gives

$$
\begin{aligned}
& e_{1, n}^{ \pm}=\frac{1}{2}\left(\begin{array}{c}
\frac{e_{n}}{ \pm i \mu_{n}} \\
e_{n} \\
\frac{e_{n}}{ \pm i \mu_{n}} \\
e_{n}
\end{array}\right)+O\left(\mu_{n}^{2 \gamma+1}\right)+O\left(\frac{1}{\mu_{n}^{2}}\right), \\
& e_{2, n}^{ \pm}=\frac{1}{2}\left(\begin{array}{c}
\frac{e_{n}}{\mp i \mu_{n}} \\
-e_{n} \\
\frac{e_{n}}{ \pm i \mu_{n}} \\
e_{n}
\end{array}\right)+O\left(\mu_{n}^{2 \gamma+1}\right)+O\left(\frac{1}{\mu_{n}^{2}}\right),
\end{aligned}
$$

For $2 \gamma+1=0$, using (3.14)-(3.15), we have

$$
\begin{aligned}
& \left(\lambda_{1, n}^{ \pm}\right)^{2}+\mu_{n}^{2}=\mp i a_{ \pm}+O\left(\frac{1}{\mu_{n}^{2}}\right), \quad \frac{1}{\lambda_{1, n}^{ \pm}}=\frac{1}{ \pm i \mu_{n}}+O\left(\frac{1}{\mu_{n}^{3}}\right), \\
& \left(\lambda_{2, n}^{ \pm}\right)^{2}+\mu_{n}^{2}=\mp i a_{\mp}+O\left(\frac{1}{\mu_{n}^{2}}\right), \quad \frac{1}{\lambda_{2, n}^{ \pm}}=\frac{1}{ \pm i \mu_{n}}+O\left(\frac{1}{\mu_{n}^{3}}\right) .
\end{aligned}
$$

Inserting (3.59)-(3.60) into (3.37) gives

$$
\begin{gathered}
e_{1, n}^{ \pm}=\sqrt{a}_{ \pm}\left(\begin{array}{c}
\frac{e_{n}}{ \pm i \mu_{n}} \\
e_{n} \\
-\frac{a e_{n}}{a_{ \pm} \mu_{n}} \\
\frac{a e_{n}}{ \pm i a_{ \pm}}
\end{array}\right)+O\left(\frac{1}{\mu_{n}^{2}}\right), \\
e_{2, n}^{ \pm}=\sqrt{a}_{\mp}\left(\begin{array}{c}
\frac{e_{n}}{ \pm i \mu_{n}} \\
e_{n} \\
-\frac{a e_{n}}{a_{\mp} \mu_{n}} \\
\frac{a e_{n}}{ \pm i a_{\mp}}
\end{array}\right)+O\left(\frac{1}{\mu_{n}^{2}}\right) .
\end{gathered}
$$

The proof is thus complete.

Remark 3.1. If $2 \gamma+1>0$, the leading term of the eigenvectors $e_{1, n}^{ \pm}$and $e_{2, n}^{ \pm}$are decoupled. The system (3.2)-(3.3) is over-damped and "essentially 
decoupled". While if $2 \gamma+1<0$, the leading term of $e_{1, n}^{ \pm}$and $e_{2, n}^{ \pm}$are well well coupled. The system (3.2)-(3.3) is right-damped in that case. In particular, when $2 \gamma+1=0$ the parameter $a$ appears explicitly in the leading term of the matrix $L_{n}$.

Lemma 3.1. Let $\left\{X_{n}\right\}_{n \geq 1}$ be a Riesz basis of subspaces in a Hilbert space $X$ and $\left\{Y_{n}\right\}_{n \geq 1}$ a Riesz sequence of subspace in $X$. Assume that there exist a sequence of isomorphisms $\left\{L_{n}\right\}_{n \geq 1}$ from $X_{n}$ onto $Y_{n}$ and positive constants $m>0, M>0$ independent of $n$ such that

$$
m\left\|x_{n}\right\| \leq\left\|L_{n} x_{n}\right\| \leq M\left\|x_{n}\right\|, \quad \forall x_{n} \in X_{n}, \quad \forall n \geq 1
$$

Assume furthermore that for each $n \geq 1$, there exists a Riesz basis $\left\{f_{n, i}\right\}_{1 \leq l \leq I_{n}}\left(I_{n} \leq+\infty\right)$ in $X_{n}$ and positive constants $c>0, C>0$ independent of $n$ such that

$$
c \sum_{i=1}^{I_{n}}\left|\alpha_{n, i}\right|^{2} \leq\left\|x_{n}\right\|^{2} \leq C \sum_{i=1}^{I_{n}}\left|\alpha_{n, i}\right|^{2}, \quad \forall x_{n}=\sum_{i=1}^{I_{n}} \alpha_{n, i} f_{n, i} .
$$

Then the sequence

$$
g_{n, i}=L_{n} f_{n, i}, \quad \forall n \geq 1, \quad 1 \leq i \leq I_{n}
$$

forms a Riesz basis in $X$.

Proof. Since $\left\{X_{n}\right\}_{n \geq 1}$ is a Riesz basis of subspaces in $X$, then for any $x \in X$ there exists a unique sequence $\left\{x_{n}\right\}_{n \geq 1}$ with $x_{n} \in X_{n}$ such that

$$
x=\sum_{n=1}^{\infty} x_{n}, \quad c^{\prime} \sum_{n=1}^{\infty}\left\|x_{n}\right\|^{2} \leq\|x\|^{2} \leq C^{\prime} \sum_{n=1}^{\infty}\left\|x_{n}\right\|^{2} .
$$

This combining with (3.64) imply that the sequence $\left\{f_{n, i}\right\}_{n \geq 1,1 \leq i \leq I_{n}}$ forms a Riesz basis in $X$.

Now define the application $L$ in $X$ as following

$$
L x=\sum_{n=1}^{\infty} L_{n} x_{n}, \quad x=\sum_{n=1}^{\infty} x_{n}
$$

It is obvious that $L$ is a linear application in $X$. Moreover, since $\left\{Y_{n}\right\}_{n \geq 1}$ is a Riesz sequence of subspaces in $X$, we get

$$
c^{\prime \prime} \sum_{n=1}^{\infty}\left\|L_{n} x_{n}\right\|^{2} \leq\|L x\|^{2} \leq C^{\prime \prime} \sum_{n=1}^{\infty}\left\|L_{n} x_{n}\right\|^{2} .
$$


Combining (3.63), (3.66) and (3.68) we get

$$
\frac{m c^{\prime \prime}}{C^{\prime}}\|x\|^{2} \leq\|L x\|^{2} \leq \frac{M C^{\prime \prime}}{c^{\prime}}\|x\|^{2}, \quad \forall x \in X
$$

It follows that $L$ is an isomorphism in $X$. Thus the sequence $\left\{g_{n, i}\right\}_{n \geq 1,1 \leq i \leq I_{n}}$, being the image of the Riesz basis $\left\{f_{n, i}\right\}_{n \geq 1,1 \leq i \leq I_{n}}$ by the isomorphism $L$, forms a Riesz basis in $X$. This achieves the proof.

Proposition 3.3. Let $\gamma \leq 0$ and $a$ be a real number small enough. Then the system of eigenvectors $\left\{e_{1, n}^{+}, e_{2, n}^{+}, e_{2, n}^{-}, e_{2, n}^{-}\right\}_{n \geq 1}$ of $\mathcal{A}$ forms a Riesz basis in $\mathcal{H}$. In particular, all eigenvectors of $\mathcal{A}$ are of the form (3.7).

Proof. For all $n \geq 1$ let

$$
W_{n}=\operatorname{Sp}\left\{E_{1, n}^{+}, E_{2, n}^{+}, E_{2, n}^{-}, E_{1, n}^{-}\right\}
$$

It is clear that $\left\{W_{n}\right\}_{n \geq 1}$ forms a Hilbert basis of subspaces and $\left\{V_{n}\right\}_{n \geq 1}$ is a Hilbert sequence of subspaces in $\mathcal{H}$. The condition (3.64) is trivial since $E_{1, n}^{+}, E_{2, n}^{+}, E_{2, n}^{-}, E_{2, n}^{-}$is a Hilbert basis in the subspace $W_{n}$. Let $L_{n}$ be defined as in (3.46). Following Proposition $3.2, L_{n}$ has a constant leading term which is invertible. This together with the fact that $L_{n}$ is invertible for all $n \geq 1$ imply the condition (3.63). Then applying Lemma 3.1, we get that the system of eigenvectors $\left\{e_{1, n}^{+}, e_{2, n}^{+}, e_{2, n}^{-}, e_{2, n}^{-}\right\}_{n \geq 1}$ forms a Riesz basis in $\mathcal{H}$. In particular, all eigenvectors of $\mathcal{A}$ are of the form (3.7). The proof is thus completed.

Remark 3.2. There were many papers based on Riesz approach for which the essential part is to show that the sequence of the root vectors is quadratically close to known Riesz basis. This approach requires a very long calculation and is limited to one-dimensional problems (see [7] and the successions).

The idea of Proposition 3.3 lies in constructing the pairwise orthogonal subspaces $V_{n}$ without the eigenvectors $e_{1, n}^{+}, e_{2, n}^{+}, e_{1, n}^{-}, e_{2, n}^{-}$being orthogonal. By this way, we reduced the quadratical convergence to the Parseval equality. In order to get the condition (3.64), we need only some asymptotic expansions such as (3.47)-(3.49) which do not give any quadratical convergence.

To fix the idea, we consider the case where $A:=-\Delta$ is the Laplacian on a bounded open set $\Omega \subset \mathbb{R}^{N}$ with the homogeneous Dirichlet boundary condition as in Example 4.1. Following a classical result of Agmon (Theorem 14.6 in [2]), we know that $\mu_{n} \sim n^{1 / N}$. Let $2 \gamma+1>0$, then the series of general term

$$
\left\|e_{k, n}^{ \pm}-E_{k, n}^{ \pm}\right\|=O\left(\frac{1}{n^{(2 \gamma+1) / N}}\right)
$$


converges in $l^{2}$ if and only if $N<2(2 \gamma+1)$. This is never true if $-1 / 2<\gamma \leq-1 / 4$ even in one-dimension. In the best case $\gamma=0$ which corresponds to the usual damping $y_{t}$, the series of general term (3.71) converges in $l^{2}$ if and only if $N<2$. This confirms that the quadratical convergence could be expected only in one-dimension.

Theorem 3.1. Let $\gamma \leq 0$ and $a$ be a real number small enough. Then for all $y_{0}, z_{0} \in D(A)$ and $y_{1}, u_{1} \in D\left(A^{\frac{1}{2}}\right)$ the energy of the system (3.2)-(3.3) has the following polynomial decay rate

$$
E(t) \leq C\left(\left\|A y_{0}\right\|_{H}^{2}+\left\|A u_{0}\right\|_{H}^{2}+\left\|A^{\frac{1}{2}} y_{1}\right\|_{H}^{2}+\left\|A^{\frac{1}{2}} u_{1}\right\|_{H}^{2}\right) \frac{1}{t^{\delta(\gamma)}}, \quad \forall t>0
$$

where

$$
\delta(\gamma)= \begin{cases}\frac{1}{\gamma+1}, & 2 \gamma+1>0 \\ -\frac{1}{\gamma}, & 2 \gamma+1<0 \\ 2, & 2 \gamma+1=0\end{cases}
$$

Proof. Following Proposition 3.1, the eigenvalues of $\mathcal{A}$ satisfy the asymptotic expansions (2.1) with

$$
\begin{cases}\alpha_{1}=-2 \gamma, \quad \alpha_{2}=2(\gamma+1), & \delta=\frac{1}{\gamma+1}, \quad 2 \gamma+1>0 \\ \alpha_{1}=\alpha_{2}=-2 \gamma, & \delta=\frac{1}{-\gamma}, \quad 2 \gamma+1<0 \\ \alpha_{1}=\alpha_{2}=1, & \delta=2, \quad 2 \gamma+1=0\end{cases}
$$

On the other hand, following Proposition 3.3 the system of eigenvectors of $\mathcal{A}$ forms a Riesz basis in $\mathcal{H}$. Then applying Theorem 2.1, we get the polynomial energy decay rate (3.72)-(3.73). The proof is thus achieved.

The following theorem gives the repartition of energy within the two equations of the system (3.2) -(3.3).

Theorem 3.2. Assume the same conditions as in Theorem 3.1. Let $w_{0} \in D(\mathcal{A})$ be an initial data and

$$
\begin{aligned}
& E_{1}(t)=\frac{1}{2}\left(\left\|A^{\frac{1}{2}} y(t)\right\|_{H}^{2}+\|z(t)\|_{H}^{2}\right), \\
& E_{2}(t)=\frac{1}{2}\left(\left\|\left.A^{\frac{1}{2}} u(t)\right|_{H} ^{2}+\right\| v(t) \|_{H}^{2}\right) .
\end{aligned}
$$

be the corresponding energy of the first equation, respectively the second equation. Then the following estimates hold

$$
\begin{array}{ll}
E_{1}(t) \leq C\left\|\mathcal{A} w_{0}\right\|^{2} \frac{1}{t^{2}}, & E_{2}(t) \leq C\left\|\mathcal{A} w_{0}\right\|^{2} \frac{1}{t^{\frac{1}{\gamma+1}}}, \quad 2 \gamma+1>0 \\
E_{1}(t) \leq C\left\|\mathcal{A} w_{0}\right\|^{2} \frac{1}{t^{\frac{1}{-\gamma}}}, & E_{2}(t) \leq C\left\|\mathcal{A} w_{0}\right\|^{2} \frac{1}{t^{\frac{1}{-\gamma}}}, \quad 2 \gamma+1 \leq 0 .
\end{array}
$$


Proof. Developing $e_{1, n}^{ \pm}, e_{2, n}^{ \pm}$on the basis $E_{1, n}^{ \pm}, E_{2, n}^{ \pm}$, we get

$$
e_{1, n}^{ \pm}=\alpha_{1, n}^{ \pm} E_{1, n}^{ \pm}+\alpha_{2, n}^{ \pm} E_{2, n}^{ \pm}, \quad e_{2, n}^{ \pm}=\beta_{1, n}^{ \pm} E_{1, n}^{ \pm}+\beta_{2, n}^{ \pm} E_{2, n}^{ \pm} .
$$

Writing

$$
w_{0}=\sum_{n=1}^{\infty} a_{1, n}^{ \pm} e_{1, n}^{ \pm}+\sum_{n=1}^{\infty} a_{2, n}^{ \pm} e_{2, n}^{ \pm}
$$

then we have

$$
\begin{aligned}
w(t) & =\sum_{n=1}^{\infty} a_{1, n}^{ \pm} e^{\lambda_{1, n}^{ \pm} t} e_{1, n}^{ \pm}+\sum_{n=1}^{\infty} a_{2, n}^{ \pm} e^{\lambda_{2, n}^{ \pm} t} e_{2, n}^{ \pm} \\
& =\sum_{n=1}^{\infty}\left(a_{1, n}^{ \pm} \alpha_{1, n}^{ \pm} e^{\lambda_{1, n}^{ \pm} t}+a_{2, n}^{ \pm} \beta_{1, n}^{ \pm} e^{\lambda_{2, n}^{ \pm}}\right) E_{1, n}^{ \pm} \\
& +\sum_{n=1}^{\infty}\left(a_{1, n}^{ \pm} \alpha_{2, n}^{ \pm} e^{\lambda_{1, n}^{ \pm} t}+a_{2, n}^{ \pm} \beta_{2, n}^{ \pm} e^{\lambda_{2, n}^{ \pm}}\right) E_{2, n}^{ \pm}
\end{aligned}
$$

The orthogonality of $E_{1, n}^{ \pm}, E_{2, n}^{ \pm}$implies

$$
\begin{aligned}
& E_{1}(t)=\frac{1}{2} \sum_{n=1}^{\infty}\left|a_{1, n}^{ \pm} \alpha_{1, n}^{ \pm} e^{\lambda_{1, n}^{ \pm} t}+a_{2, n}^{ \pm} \beta_{1, n}^{ \pm} e^{\lambda_{2, n}^{ \pm} t}\right|^{2} \\
& E_{2}(t)=\frac{1}{2} \sum_{n=1}^{\infty}\left|a_{1, n}^{ \pm} \alpha_{2, n}^{ \pm} e^{\lambda_{1, n}^{ \pm} t}+a_{2, n}^{ \pm} \beta_{2, n}^{ \pm} e^{\lambda_{2, n}^{ \pm} t}\right|^{2}
\end{aligned}
$$

If $2 \gamma+1>0$, then from (3.47) we get

$$
\alpha_{1, n}^{ \pm}=O(1), \quad \alpha_{2, n}^{ \pm}=O\left(\frac{1}{\mu_{n}^{2 \gamma+1}}\right), \quad \beta_{1, n}^{ \pm}=O\left(\frac{1}{\mu_{n}^{2 \gamma+1}}\right), \quad \beta_{2, n}^{ \pm}=O(1)
$$

Inserting (3.84) into (3.82)-(3.83), we get

$$
\begin{aligned}
& E_{1}(t) \leq C \sum_{n=1}^{\infty}\left(\frac{\left|\mu_{n} a_{1, n}^{ \pm}\right|^{2}}{\mu_{n}^{2} e^{\mu_{n}^{2 \gamma} t}}+\frac{\left|\mu_{n} a_{2, n}^{ \pm}\right|^{2}}{\mu_{n}^{4 \gamma+4} e^{\frac{a^{2} t}{\mu_{n}^{2 \gamma+2}}}}\right) \\
& E_{2}(t) \leq C \sum_{n=1}^{\infty} \frac{\left|\mu_{n} a_{1, n}^{ \pm}\right|^{2}}{\mu_{n}^{4 \gamma+4} e^{\mu_{n}^{2 \gamma} t}}+\frac{\left|\mu_{n} a_{2, n}^{ \pm}\right|^{2}}{\mu_{n}^{2} e^{\frac{a^{2} t}{\mu_{n}^{2 \gamma+2}}}} .
\end{aligned}
$$

Using the estimate for any $\theta>0, \alpha>0$

$$
\mu_{n}^{2 \theta} e^{\frac{t}{\mu_{n}^{\alpha}}} \geq C t^{\frac{2 \theta}{\alpha}}
$$


in (3.85)-(3.86), it follows that

$$
\begin{aligned}
& E_{1}(t) \leq C\left\|\mathcal{A} w_{0}\right\|^{2}\left(\frac{1}{t^{\frac{1}{-\gamma}}}+\frac{1}{t^{2}}\right) \leq C\left\|\mathcal{A} w_{0}\right\|^{2} \frac{1}{t^{2}} \\
& E_{2}(t) \leq C\left\|\mathcal{A} w_{0}\right\|^{2}\left(\frac{1}{t^{\frac{2(\gamma+1)}{-\gamma}}}+\frac{1}{t^{\frac{1}{\gamma+1}}}\right) \leq C\left\|\mathcal{A} w_{0}\right\|^{2} \frac{1}{t^{\frac{1}{\gamma+1}}}
\end{aligned}
$$

If $2 \gamma+1 \leq 0$, then from (3.48)-(3.49) we get

$$
\alpha_{1, n}^{ \pm}=O(1), \quad \alpha_{2, n}^{ \pm}=O(1), \quad \beta_{1, n}^{ \pm}=O(1), \quad \beta_{2, n}^{ \pm}=O(1) .
$$

Inserting (3.90) into (3.82)-(3.83) we get

$$
E_{1,2}(t) \leq C \sum_{n=1}^{\infty}\left(\left|\mu_{n} a_{1, n}^{ \pm}\right|^{2}+\left|\mu_{n} a_{2, n}^{ \pm}\right|^{2}\right) \frac{1}{\mu_{n}^{2} e^{\mu_{n}^{2 \gamma} t}}
$$

Then using (3.87) into (3.91) gives we get (3.78). The proof is thus complete.

Comments on theorems 3.1 and 3.2. From (3.73) we see that the decay rate $\delta(\gamma)$ is an increasing function for $-\infty<\gamma \leq-1 / 2$, decreasing for $-1 / 2 \leq \gamma \leq 0$ and achieves the maximum 2 at $\gamma=-1 / 2$. The best decay rate $\delta=2$ occurs for $\gamma=-1 / 2$.

If $2 \gamma+1>0$, the system (3.2)-(3.3) is over-damped in the sense that the eigenvectors $e_{1, n}^{ \pm}$are asymptotically decoupled from the eigenvectors $e_{2, n}^{ \pm}$. In that case, the interferences between the two equations are very weak and the wave propagates almost independently in the two equations. The energy of the first equation $E_{1}(t)$ decays more quickly than the energy $E_{2}(t)$ of the second equation.

If $2 \gamma+1 \leq 0$, the system (3.2)-(3.3) is right-damped in the sense that the eigenvectors $e_{1, n}^{ \pm}$are involved with the eigenvectors $e_{2, n}^{ \pm}$. In that case the damping applied to the first equation is well transmitted to the second equation and the system is really coupled. Consequently, the energies $E_{1}(t), E_{2}(t)$ of the two equations decay at the same rate. This balanced repartition of energies within the two equations is due to the compensation of real parts of the two branches of the eigenvalues.

A stronger damping $A^{\gamma} y_{t}$ does not necessarily give a better total decay rate of energy. A good damping should provide a compensation of the real parts of the two branches of eigenvalues and carry the transmission of the damping from one equation to another. This can be done by means of suitably weaker damping. 
We believe that the spectrum compensation is a natural phenomenon for partially damped distributed systems. It seems interesting to consider coupled systems with different operators $A_{1}, A_{2}$ as in [4]. Indeed the same results could obtained without essential difficulty in the case $A_{2}=A_{1}^{2}$ or other similar situations(see [1], [3] for example). But in general we can no long calculate explicitly the eigenvalues as in Proposition 3.1.

$\S 4$. Examples of application. Let $\Omega \subset \mathbb{R}^{n}$ a bounded open set with smooth boundary $\Gamma$. We denote by $\|\cdot\|_{0, \Omega},\|\cdot\|_{1, \Omega},\|\cdot\|_{2, \Omega} \cdots$ the norms of the Sobolev's spaces $L^{2}(\Omega), H^{1}(\Omega), H^{2}(\Omega) \cdots$ respectively.

Example 4.1. Let us consider the system of weakly coupled wave equations

$$
\left\{\begin{array}{lc}
y_{t t}-\Delta y+(-\Delta)^{-1 / 2} y_{t}+a u=0 & \text { in } \Omega, \\
u_{t t}-\Delta u+a y=0 & \text { in } \Omega, \\
y=u=0 & \text { on } \Gamma
\end{array}\right.
$$

where $a \in \mathbb{R}$ is small enough, and where $(-\Delta)^{-1 / 2}$ is a linear continuous operator from $L^{2}(\Omega)$ onto $H_{0}^{1}(\Omega)$.

Defining the operator $A$ in $L^{2}(\Omega)$ by

$$
A=-\Delta \quad \text { with } \quad D(A)=H^{2}(\Omega) \cap H_{0}^{1}(\Omega),
$$

we easily check that $A$ is a densely defined closed self-adjoint operator with compact resolvent in $L^{2}(\Omega)$. Then applying Theorem 3.1 we get the following optimal polynomial energy decay rate

$$
\begin{aligned}
& \|y(t)\|_{1, \Omega}^{2}+\left\|y_{t}(t)\right\|_{0, \Omega}^{2}+\|u(t)\|_{1, \Omega}^{2}+\left\|u_{t}(t)\right\|_{0, \Omega}^{2} \\
\leq & \frac{c}{t^{2}}\left(\left\|y_{0}\right\|_{2, \Omega}^{2}+\left\|z_{0}\right\|_{1, \Omega}^{2}+\left\|u_{0}\right\|_{2, \Omega}^{2}+\left\|v_{0}\right\|_{1, \Omega}^{2}\right)
\end{aligned}
$$

for all smooth initial data

$$
\begin{cases}y(x, 0)=y_{0}(x) \in H^{2}(\Omega) \cap H_{0}^{1}(\Omega), & y_{t}(x, 0)=z_{0}(x) \in H_{0}^{1}(\Omega), \\ u(x, 0)=u_{0}(x) \in H^{2}(\Omega) \cap H_{0}^{1}(\Omega), & u_{t}(x, 0)=v_{0}(x) \in H_{0}^{1}(\Omega),\end{cases}
$$

If we have taken a stronger damping $y_{t}$ as it was done in [4], instead of $1 / t^{2}$ we could only get $1 / t$ as the energy decay rate for the same initial data.

Example 4.2. Now we consider the system of weakly coupled plate equations

$$
\left\{\begin{array}{lr}
y_{t t}+\Delta^{2} y+\left(\Delta^{2}\right)^{-1 / 2} y_{t}+a u=0 & \text { in } \quad \Omega, \\
u_{t t}+\Delta^{2} u+a y=0 & \text { in } \quad \Omega, \\
y=\frac{\partial y}{\partial n}=u=\frac{\partial u}{\partial n}=0 & \text { on } \quad \Gamma
\end{array}\right.
$$


where $a \in \mathbb{R}$ is small enough, and where $\left(\Delta^{2}\right)^{-1 / 2}$ is a linear continuous operator from $L^{2}(\Omega)$ onto $H_{0}^{2}(\Omega)$.

Defining the operator $A$ in $L^{2}(\Omega)$ by

$$
A=\Delta^{2} \quad \text { with } \quad D(A)=H^{4}(\Omega) \cap H_{0}^{2}(\Omega)
$$

we check easily all the conditions of Theorem 3.1. Then for any smooth initial data

$$
\begin{cases}y(x, 0)=y_{0}(x) \in H^{4}(\Omega) \cap H_{0}^{2}(\Omega), & y_{t}(x, 0)=z_{0}(x) \in H_{0}^{2}(\Omega) \\ u(x, 0)=u_{0}(x) \in H^{4}(\Omega) \cap H_{0}^{2}(\Omega), & u_{t}(x, 0)=v_{0}(x) \in H_{0}^{2}(\Omega)\end{cases}
$$

we have

$$
\begin{aligned}
& \|y(t)\|_{2, \Omega}^{2}+\left\|y_{t}(t)\right\|_{0, \Omega}^{2}+\|u(t)\|_{2, \Omega}^{2}+\left\|u_{t}(t)\right\|_{0, \Omega}^{2} \\
\leq & \frac{c}{t^{2}}\left(\left\|y_{0}\right\|_{4, \Omega}^{2}+\left\|z_{0}\right\|_{2, \Omega}^{2}+\left\|u_{0}\right\|_{4, \Omega}^{2}+\left\|v_{0}\right\|_{2, \Omega}^{2}\right) .
\end{aligned}
$$

Similarly if we have taken a stronger feedback control $y_{t}$ as it was done in [4], we could only get $1 / t$ as the energy decay rate for the same initial data.

In [4] more general systems with different operator $A_{1}, A_{2}$ were considered under an artificial condition $D\left(A_{2}^{j / 2}\right) \subset D\left(A_{1}\right), j \geq 2$ which restricts the applications to the distributed systems of the same kind boundary conditions (see examples 6.1-6.5 in [4]). The following example shows that the above mentioned condition is not necessary and the polynomial energy decay rate should be true for more general coupled systems.

Example 4.3. Consider the following system of compactly coupled and partially damped Euler-Bernoulli beam equations

$$
\left\{\begin{array}{l}
y_{t t}+y_{x x x x}+b y_{t}+a u_{x}+y=0, \quad 0<x<\pi \\
u_{t t}+u_{x x x x}-a y_{x}=0, \quad 0<x<\pi \\
y_{x}(0)=y_{x x x}(0)=y_{x}(\pi)=y_{x x x}(\pi)=0 \\
u(0)=u_{x x}(0)=u(\pi)=u_{x x}(\pi)=0 .
\end{array}\right.
$$

Putting

$V=\left\{y \in H^{2}(0, \pi), y^{\prime}(0)=y^{\prime}(\pi)=0\right\}, \quad W=\left\{u \in H^{2}(0, \pi), u(0)=u(\pi)=0\right\}$

we define the energy space

$$
\mathcal{H}=V \times L^{2}(0, \pi) \times W \times L^{2}(0, \pi)
$$


It is easy to prove that the system (4.8) generates a $C_{0}$-semigroup of contractions on the space $\mathcal{H}$ for $b>0$ and $a$ small enough. Moreover, setting the energy

$$
E(t)=\frac{1}{2} \int_{0}^{\pi}\left(|y|^{2}+\left|y_{x x}\right|^{2}+\left|y_{t}\right|^{2}+\left|u_{x x}\right|^{2}+\left|u_{t}\right|^{2}-a y_{x} \bar{u}-a u \bar{y}_{x}\right) d x
$$

we have

$$
\frac{d}{d t} E(t)=-b \int_{0}^{\pi}\left|y_{t}\right|^{2} d x \leq 0 .
$$

Now we consider the associated eigen-problem

$$
\begin{cases}\lambda^{2} y+y_{x x x x}+b \lambda y+a u_{x}+y=0 & 0<x<\pi \\ \lambda^{2} u+u_{x x x x}-a y_{x}=0 & 0<x<\pi \\ y_{x}(0)=y_{x x x}(0)=y_{x}(\pi)=y_{x x x}(\pi)=0 & \\ u(0)=u_{x x}(0)=u(\pi)=u_{x x}(\pi)=0 .\end{cases}
$$

Let

$$
y=\alpha_{n} \cos n x, \quad u=\beta_{n} \sin n x
$$

be an eigenfunction. Then $\lambda$ must satisfy the system

$$
\left\{\begin{array}{l}
\alpha_{n}\left(\lambda^{2}+b \lambda+n^{4}+1\right)-a \beta_{n} n=0 \\
\beta_{n}\left(\lambda^{2}+n^{4}\right)+\alpha_{n} a n=0
\end{array}\right.
$$

which has non trivial solution if and only if

$$
\left(\lambda^{2}+n^{4}\right)\left(\lambda^{2}+b \lambda+n^{4}+1\right)+a^{2} n^{2}=0 .
$$

Proceeding as in Proposition 3.1, we find easily that

$$
\begin{aligned}
& \lambda_{1, n}^{ \pm}= \pm i n^{2}-\frac{b}{2}+O\left(\frac{1}{n^{2}}\right) \\
& \lambda_{2, n}^{ \pm}= \pm i n^{2}-\frac{a^{2}}{2 b n^{2}}+O\left(\frac{1}{n^{6}}\right) .
\end{aligned}
$$

Then for $\lambda=\lambda_{1, n}^{ \pm}$, taking

$$
\alpha_{1, n}^{ \pm}=\frac{1}{\sqrt{2}}, \quad \beta_{1, n}^{ \pm}=-\frac{a n}{\left(\lambda_{1, n}^{ \pm}\right)^{2}+n^{4}} \alpha_{1, n}^{ \pm}=O\left(\frac{1}{n}\right)
$$

in (4.12) we get the corresponding eigenvector

$$
e_{1, n}^{ \pm}=\frac{1}{\sqrt{2}}\left(\begin{array}{c}
\frac{\cos n x}{ \pm i n^{2}} \\
\cos n x \\
0 \\
0
\end{array}\right)+O\left(\frac{1}{n}\right), \quad \forall n \geq 1
$$


For $\lambda=\lambda_{2, n}^{ \pm}$, taking

$$
\beta_{2, n}^{ \pm}=\frac{1}{\sqrt{2}}, \quad \alpha_{1, n}^{ \pm}=-\frac{\left(\lambda_{1, n}^{ \pm}\right)^{2}+n^{4}}{a n} \beta_{2, n}^{ \pm}=O\left(\frac{1}{n}\right)
$$

in (4.12) we get the corresponding eigenvector

$$
e_{2, n}^{ \pm}=\frac{1}{\sqrt{2}}\left(\begin{array}{c}
0 \\
0 \\
\frac{\sin n \pi x}{ \pm i n^{2}} \\
\sin n \pi x
\end{array}\right)+O\left(\frac{1}{n}\right), \quad \forall n \geq 1
$$

Using the same procedure as in Proposition 3.3, we can prove that the system $\left\{e_{1, n}^{ \pm}, e_{2, n}^{ \pm}\right\}_{n \geq 1}$ form a Riesz basis in $\mathcal{H}$. But in the one-dimensional case, the Riesz basis property is evident because the eigenvectors $e_{1, n}^{ \pm}, e_{2, n}^{ \pm}$are quadratically closed to a Hilbert basis due to the expansions (4.17)-(4.18), therefore form a Riesz basis in $\mathcal{H}$ (Theorem 4.2.3 in [6] and Theorem 4.1 in [19] for the original idea on the eigenvalues of lower frequence). Moreover, (4.15)(4.16) show that the eigenvalues of the system (4.11) satisfy the condition (2.1) with $\mu_{1, n}=\mu_{2, n}=n^{2}$ and $\alpha_{1}=0, \alpha_{2}=1$. Then applying Theorem 2.1, we get the following polynomial energy decay rate

$$
E(t) \leq C\left(\left\|y_{0}\right\|_{4}^{2}+\left\|z_{0}\right\|_{2}^{2}+\left\|u_{0}\right\|_{4}^{2}+\left\|v_{0}\right\|_{2}^{2}\right) \frac{1}{t^{2}}, \quad \forall t>0
$$

for the solution of the system (4.8) with smooth initial data $y_{0} \in H^{4}(0, \pi) \cap$ $V, u_{0} \in H^{4}(0, \pi) \cap W$ and $z_{0} \in V, v_{0} \in W$.

Remark 4.1. The coupling terms $a y_{x}, a u_{x}$ do not appear in the expression (4.10). Nevertheless the energy decays at the rate $1 / t^{2}$ instead of $1 / t$ for the system coupled through the displacements ay, au. 


\section{References}

[1] M. Afilal and F. Ammar Khodja, Stability of coupled second order equations, Comput. Appl. Math. 19(2000), 91-107.

[2] S. Agmon, Lectures on elliptic boundary values problems, Van Nostrand, 1965.

[3] F. Ammar Khodja and A. Benabdallah, Sufficient conditions for uniform stabilization of second order equations by dynamical controllers, Dynam. Contin. Discrete Impuls. Systems 7(2000), 207-222.

[4] F. Alabau, P. Cannarsa and V. Komornik, Indirect internal stabilization of weakly coupled evolution equations, J. Evolution Equations 2 (2002), 127-150.

[5] A. Bátkai, K.-J. Engel, J. Prüss and R. Schnaubelt, Polynomial stability of operator semigroups, to appear in Math. Nachr.

[6] I. C. Gohberg and M.G. Krein, Introduction to the theory of linear non selfadjoint operators, AMS, Providence, 1969.

[7] B.Z. Guo Riesz basis approach to the stabilization of a flexible beam with a tip mass, SIAM J. Control Optim. 39(2001), 1736-1747.

[8] F. Huang, Characteristic condition for exponential stability of linear dynamical systems in Hilbert spaces, Chinese Ann. of Diff. Eqs, 1(1985), 43-56.

[9] S. Jaffard, M. Tucsnak and E. Zuazua, Singular inter stabilization of the wave equation, J. Diff. Equa., 45(1998), 184-215.

[10] W. Littman and B.Liu, On the spectral properties and stabilization of acoustic flow, SIAM J. Appl. Math. 59(1999), 17-34

[11] W. Littman and L. Markus, Some recent results on control and stabilization of flexible structures, Proc. COMCON Workshop, Montpelier (1987).

[12] J.-L. Lions, Contrôlabilité exacte et stabilisation de systèmes distribués, Masson, Paris, 1984.

[13] Z. Liu and B.P. Rao, Characterization of polynomial decay rate for the solution of linear evolution equation, to appear in Z. Angew. Math. Phys.

[14] K. Liu, Z. Liu and B. Rao, Exponential stability of an abstract non-dissipative linear system, J. SIAM Contr. Optim. 40(2001), 149-165.

[15] Z. Liu and S. Zheng, Semigroup associated with dissipative systems, Chapman \& Hall/CRC Research Notes in Mathematics, 394. Chapman \& Hall/CRC, Boca Raton, 1999.

[16] P. Loreti and B. Rao, Compensation spectrale et taux de décroissance optimal de l'energie de systèmes partiellement amortis, C. R. Acad. Sci. Paris, 337, Série I (2003), 531-536

[17] A. Pazy, Semigroups of Linear Operators and Applications to Partial Differential Equations, Springer, New York, 1984.

[18] J. Prüss, On the spectrum of $C_{0}$-semigroups, Trans. Amer. Math. Soc. 284 (1984), 847-857. 
[19] B. Rao, Optimal energy decay rate in the Rayleigh beam equation, Optimization Methods in Partial Differential Equations, ed. Cox and Lasiecka, Contemporary Mathematics, 209 (1997) 211-229.

[20] B. Rao, Stabilization of a plate equation with dynamical boundary control, J. SIAM Control Optim. 36(1998), 148-164.

[21] B. Rao and A. Wehbe, Polynomial energy decay rate and strong stability of Kirchhoff plates with non-compact resolvent, to appear in J. Evolution Equations.

[22] D. Russell, A general framework for the study of indirect damping mechanisms in elastic systems, J. Math. Anal. Appl. 173(1993), 339-354.

[23] A. Wehbe, Boundary stabilization of wave equations with dynamical control, Applied Mathematics Letters, 16(2003), 357-363.

[24] X, Zhang and E. Zuazua, Polynomial decay and control of a $1-d$ hyperbolicparabolic coupled system, J. Diff. Eqs. 204(2004), 380-438. 